\title{
Wearable and wireless accelerometer systems for monitoring Parkinson's disease patients-A perspective review
}

\author{
Robert LeMoyne \\ Department of Biological Sciences, Northern Arizona University, Flagstaff, USA; \\ robert.lemoyne@nau.edu, rlemoyne07@gmail.com
}

Received 10 September 2013; revised 10 October 2013; accepted 18 October 2013

Copyright (c) 2013 Robert LeMoyne. This is an open access article distributed under the Creative Commons Attribution License, which permits unrestricted use, distribution, and reproduction in any medium, provided the original work is properly cited.

\begin{abstract}
Parkinson's disease is a growing medical concern as societies, such as the United States of America, become progressively aged. Therapy strategies exist for the amelioration of Parkinson's disease symptoms, and the quantification of attributes, such as hand tremor, can provide valuable feedback. Wearable and wireless accelerometer systems for monitoring Parkinson's disease patients have been progressively advanced over the course of the past half-decade. In particular, wireless accelerometer nodes and smartphones, such as the iPhone, hold promise for optimizing therapy strategy by providing convenient quantified feedback. This perspective review addresses the current advances in wearable and wireless accelerometer systems for monitoring Parkinson's disease patients and forecasts for the near future.
\end{abstract}

Keywords: Parkinson's Disease; Wireless Accelerometer; Parkinson's Disease Tremor Quantification; Wearable Device

\section{INTRODUCTION}

Parkinson's disease generally manifests in people aged 55 years and older [1]. Approximately one million people have Parkinson's disease in the United States of America [2]. Intuitively, for societies that are aging, Parkinson's disease is an increasing medical concern. With the growing prevalence of Parkinson's disease, wearable wireless applications may ameliorate strain on limited medical resources while improving patient quality of care. Over the recent half-decade, wireless accelerometer applications have been advocated and success- fully tested and evaluated [3-7]. Wireless accelerometer systems enable the capacity to quantify and track patient status [3-5]. Devices that are wireless and wearable can be effectively applied in rural settings. Specialized resources in urban settings can diagnose the acquired quantified data. A patient's quantified data, such as Parkinson's disease tremor, can provide critical diagnostic insight for the optimization of patient-specific therapy, thereby improving quality of life [8]. The following perspective review addresses the advances in wearable and wireless accelerometer systems for Parkinson's disease over the course of the recent half-decade with implications regarding future advances.

\section{BACKGROUND OF PARKINSON'S DISEASE}

Parkinson's disease is a neurodegenerative disorder manifesting in distinct movement disorder features. Prevalent movement dysfunctions are: shuffling of gait, impairment to balance, rigidity due to amplified muscle tone, and resting tremor [2]. There are established strategies for reducing the severity of the characteristic symptoms of Parkinson's disease, such as drug therapy, deep brain stimulation, and pallidotomy [2,9,10]. Pallidotomy incorporates brain surgery that induces a lesion to the internal aspect of the globus pallidus, which is normally reserved as a final alternative [2,9]. Deep brain stimulation of the pallidal, thalamic, and substhalamic aspects of the brain have been applied to treat Parkinson's disease symptoms. However, there are literally thousands of deep brain stimulation parameter settings regarding the electrode polarity, amplitude, frequency, and pulse width [10]. Drug therapy involves prescription of conventional drugs, such as L-dopa, with the dosage based on the discretion of the clinician [2]. 


\section{WEARABLE AND WIRELESS APPLICATIONS FOR PARKINSON'S DISEASE QUANTIFICATION}

Wearable applications, such as wireless accelerometer devices, have been advocated for augmenting the clinical diagnostic acuity for optimizing a patient's unique drug therapy $[3,5,8]$. The acquisition of patient-optimized deep brain stimulator parameter settings through the quantified feedback of wireless accelerometer systems has been advocated [5]. Logically, the progressive evolution of wearable and wireless accelerometer systems may substantially improve quality of life for Parkinson's disease patients through specific and optimized therapy strategies.

For approximately the last half-decade, wearable and wireless accelerometers have advanced the treatment capabilities for people with Parkinson's disease. LeMoyne et al. during 2009 conducted preliminary proof of concept for wireless accelerometers to quantify simulated Parkinson's disease hand tremor, while also advocating the utility for wireless accelerometer strategies $[3,6]$. During the same time span, Giuffrida et al. assessed Kinesia ${ }^{\mathrm{TM}}$, which conveyed motion sensor data wirelessly through a wrist-mounted command module [11]. However, the Kinesia ${ }^{\mathrm{TM}}$ device may be prone to having the wire between the command module and sensor becoming dislodged. Wireless accelerometer configurations have been applied for the quantification of Parkinson's disease features within the scope of daily living activities [12-14].

Smartphones, such as the iPhone, enable a unique and convenient means for the quantification of Parkinson's disease hand tremor. Notably the iPhone is geometrically on the scale of the dorsum of an average human hand. Therefore the iPhone can be readily mounted by glove to the hand of a Parkinson's disease patient. The glove mounting strategy facilitates a simple and easy to reproduce mounting strategy. The iPhone is equipped with motion sensors, such as an accelerometer. With a suitable iPhone application, a recording of the acceleration waveform for Parkinson's disease tremor can be conveyed wirelessly through email. The broad coverage range enables remote setting wireless transmission of the acceleration waveform data to distant post-processing resources. LeMoyne et al. pioneered the application of the iPhone for the quantification of Parkinson's disease hand tremor [5].

During 2010, LeMoyne et al. successfully demonstrated the ability of the iPhone to acquire and record the acceleration signal for Parkinson's disease hand tremor. The experiment was conducted in the area of Pittsburgh, Pennsylvania involving a subject with and a subject without Parkinson's disease. The recorded acceleration waveform was conveyed by wireless transmission through email for post-processing in greater Los Angeles, California. The research successfully demonstrated the ability of the wireless accelerometer iPhone application to convey data to remote locations for post-processing [5]. The iPhone application is envisioned to be operated by either the patient or through the assistance of a caregiver. Kostikis et al. later in 2011 also applied a similar smartphone application for quantifying Parkinson's disease features [15].

During 2012 to 2013, LeMoyne et al. published another configuration for monitoring Parkinson's disease hand tremor with a wireless accelerometer node. Wireless signal connectivity to a local PC was established through a base station equipped with a USB connector. Engineering proof of concept was established through simulated Parkinson's disease hand tremor and contrasted to a static control. The system requirements for the wireless accelerometer configuration implicate the application as suitable to be operated by a caregiver with basic personal computer skills [7].

\section{CONCLUSION}

Future advances in software technology and hardware miniaturization are anticipated to be critical for the evolution of wireless accelerometers for quantifying Parkinson's disease attributes, such as tremor. Wireless accelerometers may be readily integrated into wearable devices. Multiple strategies are available, such as a wireless accelerometer node and an iPhone, which can be secured to the dorsum of the hand through a glove. Configurations are available that may be operated by a caregiver or only the patient. The recorded acceleration waveforms can be transmitted via wireless to remote post-processing resources. Post-processing can augment the optimization of treatment therapy. In particular, the iPhone is equipped with a robust capacity for applications. iPhone applications can be tailored to the Parkinson's disease patient's specific needs enabling optimal quality of life. For example, a Parkinson's disease patient's grandchild could develop an iPhone application uniquely adapted to the patient's health needs.

\section{REFERENCES}

[1] Seeley, R.R., Stephens, T.D. and Tate, P. (2003) Anatomy and physiology. McGraw-Hill, New York.

[2] Kandel, E.R., Schwartz, J.H. and Jessell, T.M. (2000) Principles of neural science. McGraw-Hill, New York.

[3] LeMoyne, R., Coroian, C., Mastroianni, T., Opalinski, P., Cozza, M. and Grundfest, W. (2009) The merits of artificial proprioception, with applications in biofeedback gait rehabilitation concepts and movement disorder characterization. In: Barros de Mello, C.A., Ed., Biomedical Engineering, Intech, Vienna, 165-198. 
[4] LeMoyne, R., Coroian, C., Mastroianni, T. and Grundfest, W. (2008) Accelerometers for quantification of gait and movement disorders: A perspective review. Journal of Mechanics in Medicine and Biology, 8, 137-152. http://dx.doi.org/10.1142/S0219519408002656

[5] LeMoyne, R., Mastroianni, T., Cozza, M., Coroian, C. and Grundfest, W. (2010) Implementation of an iPhone for characterizing Parkinson's disease tremor through a wireless accelerometer application. Proceeding of the 32nd Annual International Conference of the IEEE EMBS, Buenos Aires, 31 August-4 September 2010, 4954-4958.

[6] LeMoyne, R., Coroian, C. and Mastroianni, T. (2009) Quantification of Parkinson's disease characteristics using wireless accelerometers. Proceeding of the International Conference on Complex Medical Engineering (CME2009) of the IEEE/ICME, Tempe, 9-11 April 2009, 1-5.

[7] LeMoyne, R., Mastroianni, T. and Grundfest W. (2013) Wireless accelerometer configuration for monitoring Parkinson's disease hand tremor. Advances in Parkinson's Disease, 2, 62-67.

http://dx.doi.org/10.4236/apd.2013.22012

[8] Patel, S., Park, H., Bonato, P., Chan, L. and Rodgers, M. (2012) A review of wearable sensors and systems with application in rehabilitation. Journal of NeuroEngineering and Rehabilitation, 9, 1-17. http://dx.doi.org/10.1186/1743-0003-9-21

[9] Nolte, J. and Sundsten, J.W. (2002) The human brain: An introduction to its functional anatomy. Mosby, St. Louis.

[10] Volkmann, J., Moro, E. and Pahwa, R. (2006) Basic algorithms for the programming of deep brain stimulation in Parkinson's disease. Movement Disorders, 21, S284-S289. http://dx.doi.org/10.1002/mds.20961

[11] Giuffrida, J.P., Riley, D.E., Maddux B.N. and Heldman, D.A. (2009) Clinically deployable Kinesia technology for automated tremor assessment. Movement Disorders, 24, 723-730. http://dx.doi.org/10.1002/mds.22445

[12] Cancela, J., Pansera, M., Arredondo, M.T., Estrada, J.J., Pastorino, M., Pastor-Sanz, L. and Villalar, J.L. (2010) A comprehensive motor symptom monitoring and management system: The bradykinesia case. Proceeding of the 32nd Annual International Conference of the IEEE EMBS, Buenos Aires, 31 August-4 September 2010, 1008-1011.

[13] Pastorino, M., Cancela, J., Arredondo, M.T., Pansera, M., Pastor-Sanz, L., Villagra, F., Pastor, M.A. and Martin, J.A. (2011) Assessment of bradykinesia in Parkinson's disease patients through a multi-parametric system. Proceeding of the 33rd Annual International Conference of the IEEE EMBS, Boston, 30 August-3 September 2011, 1810-1813.

[14] Cancela, J., Pastorino, M., Arredondo, M.T., Pansera, M., Pastor-Sanz, L., Villagra, F., Pastor, M.A. and Gonzalez, A.P. (2011) Gait assessment in Parkinson's disease patients through a network of wearable accelerometers in unsupervised environments. Proceeding of the 33rd Annual International Conference of the IEEE EMBS, Boston, 30 August-3 September 2011, 2233-2236.

[15] Kostikis, N., Hristu-Varsakelis, D., Arnaoutoglou, M., Kotsavasiloglou, C. and Baloyiannis, S. (2011) Towards remote evaluation of movement disorders via smartphones. Proceeding of the 33rd Annual International Conference of the IEEE EMBS, Boston, 30 August-3 September 2011, 5240-5243. 